Contents List available at VOLKSON PRESS

Intelligent Computing and Information Engineering (ICIE )

DOI : http://doi.org/10.26480/icie.01.2017.48.52

Journal Homepage: : https://www.intelcomp-design.com/

\title{
BIOMECHANICAL EXPERIMENTAL STUDY ON HUMAN LIVER AND SPLEEN AND OPTIMIZATION DISCUSSION OF TISSUE FINITE ELEMENT SIMULATION
}

\author{
Qiu Jinlong 1, Wang Chunchen ${ }^{2}$, Li Kui ${ }^{1}$, Duan Aowen ${ }^{1}$, Yin Zhiyong 1* \\ ${ }^{1}$ The Third Affiliated Hospital of Third Military Medical University, Chongqing, China \\ 2PLA 93685 force, China. \\ *Corresponding author E-mail: qiujinlong@4008970100.com
}

This is an open access article distributed under the Creative Commons Attribution License, which permits unrestricted use, distribution, and reproduction in any medium, provided the original work is properly cited

\section{ARTICLE DETAILS}

\section{Article History:}

Received 12 May2017

Accepted 12 July 2017

Available online 14 September 2017

Keywords:

Damage biomechanics, liver,

spleen, finite element simulation,

optimization.

\section{ABSTRACT}

In this study, the biomechanical properties of human liver and spleen were studied by quantifying the damage mechanisms of various organs, the biomechanical response and the rate dependence of model materials. Used CIREN and NASS-CDS databases to determine the impact characteristics of liver and spleen injuries. The fresh human organs of ram type compression test and strain rate test were conducted to determine the optimum conditions for each organ of the finite element simulation. The results showed that the responses of human liver and spleen were nonlinear and rate dependent. This study will enhance the understanding of visceral organ damage, and provide a biomechanical basis for further establishing the finite element model of human internal organs.

\section{Introduction}

Car crash usually results in severe blunt abdominal trauma, where most of the injuries belong to internal organ damage [1,2]. At present, for the assessment of car collision accident injury risk crash test dummy internal general entities are not equipped with abdominal organs (viscera in vivo, and asymmetric) while airbags and seat belts and other safety restraint technology has been popularized, finite element model (Finite element models, FEMs) has been reduced collision related abdominal injury research the main tool [3-5]. Therefore, it is necessary through the biological collision experiment of human internal organs, to obtain reliable biomechanical data from the data, thus verified on the basis of the finite element model of refinement, to accurately assess the risk of injury.

Because the finite element model allowing calculations based on damage mechanical dependent physical variables (e.g., stress and strain) to predict damage, the establishment of tissue level tolerance values is crucial for accurate prediction of damage [6]. Some biomechanical studies have been conducted to assess mechanical responses and damage tolerance of internal organs by compression tests on intact animals or human cadaveric [7, 8]. Although these studies have made important contributions to organ level verification data, they are limited in their ability to quantify local stresses and strains necessary for validation of local finite element model validation.

Some studies have investigated liver failure characteristics by tensile tests of isolated samples of liver or spleen tissue [9, 10]. Although some groups have studied the factors that affect the liver and spleen parenchyma materials deeply, they still have limitations [11].

In this study, we studied the liver and spleen injury and material properties through biomechanical experiments, mainly including: it determined the collision characteristics that lead to liver and spleen damage through accident data collection and analysis; a whole pressure impact test of intact human organs was carried out; and strain and compression materials of multiple loading rates were tested on human liver and spleen parenchyma samples, to optimize the finite element material model.

\section{MATERIALS AND METHODS}

\subsection{Integrated data acquisition and analysis}

In this study, the liver and spleen were incorporated into the chest organ. The United States National Automotive sampling system / crashworthiness database (National Automotive Sampling System - Crashworthiness Data System, NASS/CDS, USA) was used to determine the thoracic organ damage distribution (only models after 1998, front passenger, front collision). Crash damage studies and Crash Injury and (The Research Network CIREN) were used to determine the collision characteristics and physical components in cases of liver or spleen injury. For each damage group, crash characteristics were measured.

\subsection{Organ and tissue tests}

First, collect and preserve adult organs (liver and spleen) under 80 years of age. Each organ was obtained within 36 hours after death and was tested within 48 hours of death to minimize the adverse effects of tissue degradation. The overall test of the organ was carried out through a double column bench test system (INSTRON, type 5960, INSTRON ®, USA) (Figure 1.a). A series of shock tests were performed on each organ. The recovery time of each organ at each test interval is about 10 minutes. Used FaroArm (FARO, Platinum, 8Ft, Arm, FARO, USA; P1 Scanner, Switzerland) scanning organs and impactor to 
obtain 3D surface geometry. The organ was perfused with heated fluid for about 1 hours before scanning. A three-dimensional surface geometry of each organ was obtained in order to develop and validate a finite element model conforming to organ specificity.

Secondly, the tensile test of tissue level was carried out. Cut a square block from the body of the liver or spleen. Used custom stamped plugs and bases to obtain specimens of bone shape tests that are normally used in uniaxial tensile tests. The main components of the tension test device were SHPB Hopkinson tie rod and compression bar system (Luoyang Levi Technology Co., Ltd., China), based on the modified loading of a customized single axle dynamic tensile device (Figure 1.b). In the test, the test system applied a tensile load to the specimen at a constant speed in the form of simultaneous separation of the top and bottom clamps at the same time. By kinematic analysis, local stress calculations are performed based on the nearest optical mark around the torn tear position.

Thirdly, the tissue level compression test was performed again. As with tension tests, liver and spleen sections of constant thickness were obtained using the same customized blade assembly and slice clamp. The main component of the compression test device was a high-speed servo hydraulic material testing system (MTS Systems Corporation, MTS-810, Eden Prairie, MN). To study rate dependence, each sample was compressed to four levels of expected strain rate at $0.01 \mathrm{~s}-1,0.1 \mathrm{~s}-1,1.0 \mathrm{~s}-1$, or $10.0 \mathrm{~s}-1$, respectively. Used of custom Matlab code analysis threshold top view image. Characteristic mean values were analyzed for each loading rate under tension and compression.

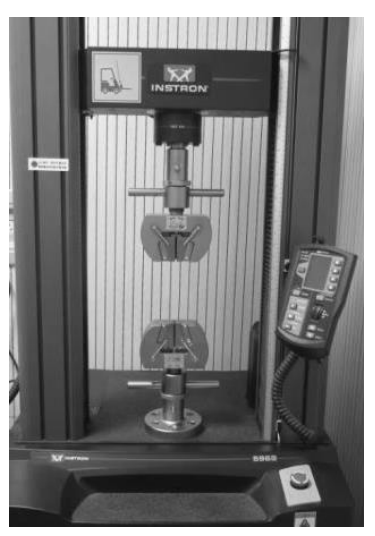

a

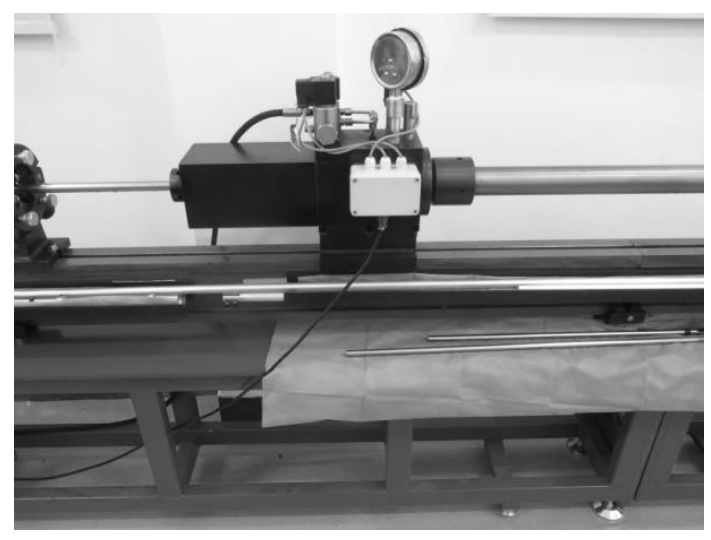

b

\subsection{Material model optimization}

Figure 1: Test devices

Loaded the original data and separating them into different strain rate categories, formed strain rate sequences, and generated two diagrams of displacement-time and force - time. Tests at constant displacement rates were performed to investigate the characteristics of the average tensile in the time dimension, and the tensile time diagrams of the strain rate sequences were obtained. Stress strain characteristics were defined for the material model. The overall stress-strain curves could be calculated by regression of the strain rate series. The different material models were compared with the target stressstrain curves to find the best model that matches the biomechanical experimental results.

\section{RESULTS AND DISCUSSION}

\subsection{Overall data analysis results}

The distribution results in Figure 2 are basically consistent with Thor's research conclusions [11]. The cases involved 80 people, including $12.5 \%$ liver injury (10 people) and $20 \%$ splenic injuries (16 persons). The collision characteristics were shown in Figure 3.

Normally, occupants of a case could have multiple anatomical injuries, so each of the injuries listed was not exclusive. It was also possible to determine the physical properties involved in each biomarker damage. Most biomarkers of liver damage were attributed to the seatbelt. Most biomarkers of splenic injury mechanics were attributed to car doors.

\subsection{Organ and tissue test results}

The scanning results of the 3D surface geometry of the organs were shown in figure 4. A sub destructive loading test for 3 perfused livers was shown in figure 5. Relative to the deflection curve, the overall force of the liver showed that the compression reaction is nonlinear and rate dependent. Tissue level tensile tests were carried out to obtain stress-strain curves of approximately $0.1 \mathrm{~S}^{-1}$ specimens, and the mean and standard deviations of the corresponding characteristics (Figure 6). The stress-strain curve showed that the tensile response is nonlinear for both the liver and spleen tensile specimens. In the current study, although the use of $1 \mathrm{~g}$ initial conditions provided strain a consistent initial state for all specimens, this situation on the tip of the stressstrain curve of impact should be resolved and quantified in future research. The tensile test of liver and spleen showed that the rate was dependent, and the stress increased with the increase of strain rate as the strain decreased (Figure 7). The results also showed that the tensile failure stress and strain of liver soft tissue were significantly higher than that of spleen.

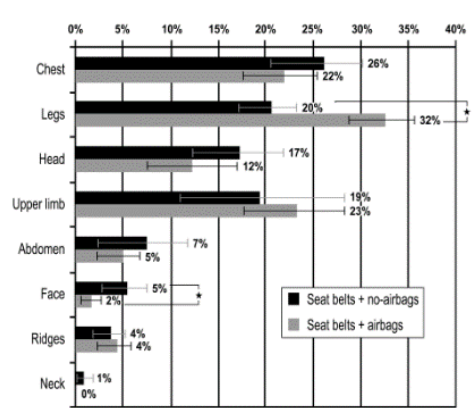

a. Human MAIS regional distribution of $3+$ damage (weighted)

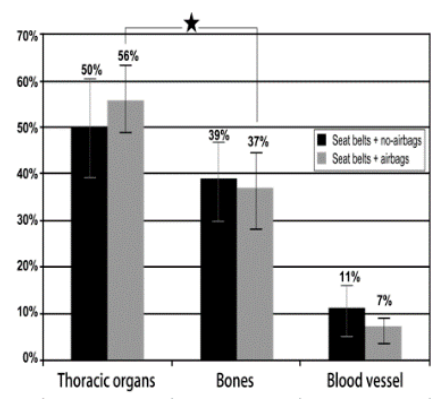

b. Tissue type profile (weighted) of chest injuries in MAIS 3+

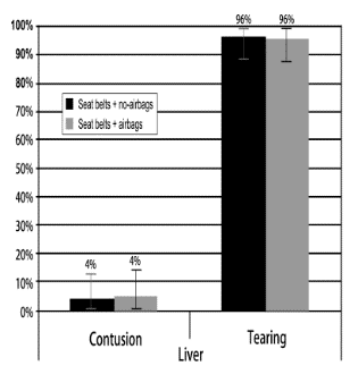

c. Distribution pattern of lesion type (weighted) in cases of AIS3 + liver injury 
Figure 2: Damage distributions

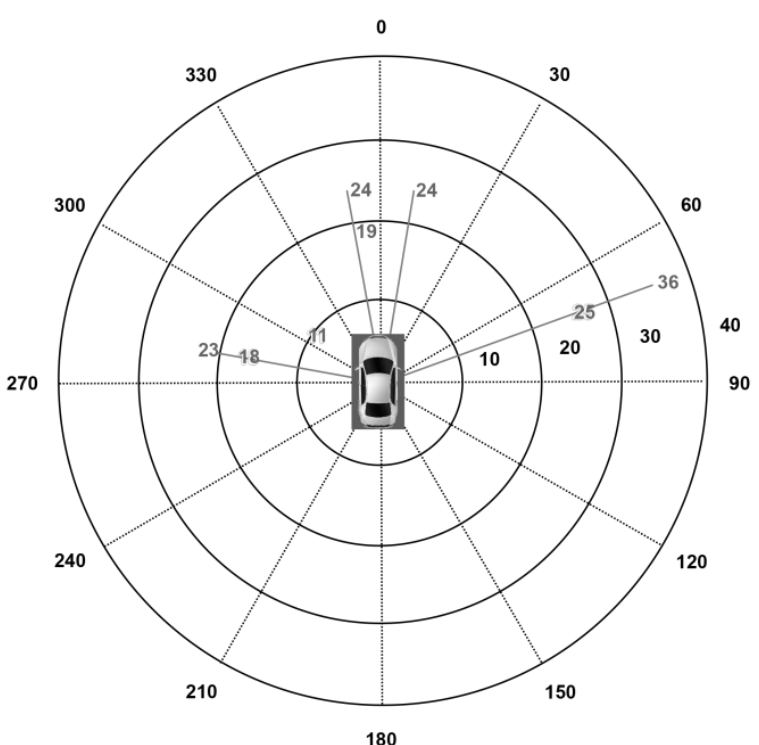

a. Radial maps of collisions Delta-V and PDOF in liver injury cases

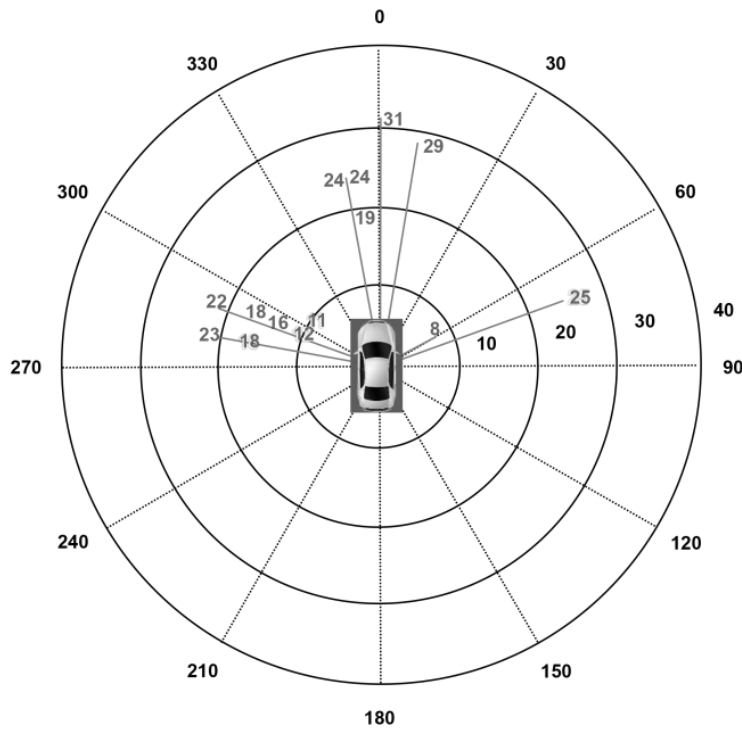

b. Radial maps of collision Delta-V and PDOF in cases of splenic injury

Figure 3: Collision characteristics

In the compression test of the tissue level, the mechanical failure position was shown in figure 8. The stress-strain curve (Figure 9) showed that the compression response is nonlinear for the liver and spleen compression samples. The results of liver and spleen compression tests showed a rate dependent, strain reduction and stress increase as the strain rate increases (Figure 10). The results also showed that the compressive stress of the liver parenchyma was significantly higher than that of the spleen.

\subsection{On the tissue level finite element simulation}

The best shape of the material model has been obtained by obtaining polynomials from the aforementioned tensile and stress normalization data. It has defined scaling parameters to modify the input curve. Using the scaling factor method, three independent loading rates have been optimized, each strain rate corresponding to a loading rate. The Multi Island Genetic Algorithm has been adopted to optimize the factor to minimize the minimum square error between the model output and the target stress relative to the strain curve.

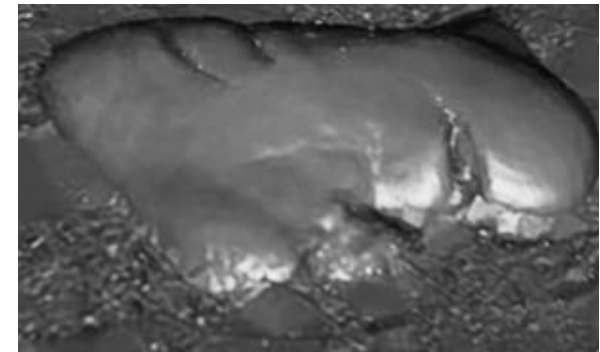

a. The liver

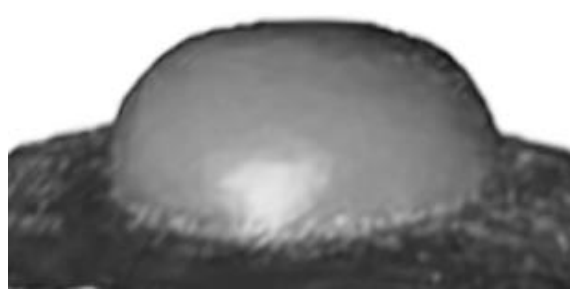

b. The spleen

Figure 4: 3D surface geometry

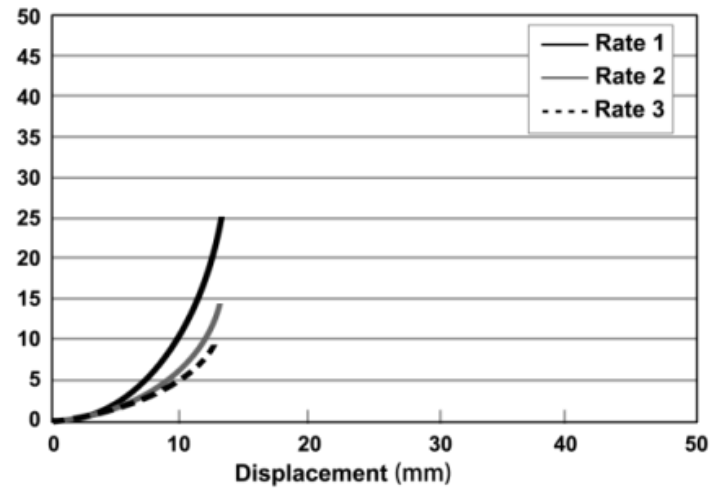

Figure 5: Liver damage loading at various loading rates (cutting data at $14 \%$ pressure)

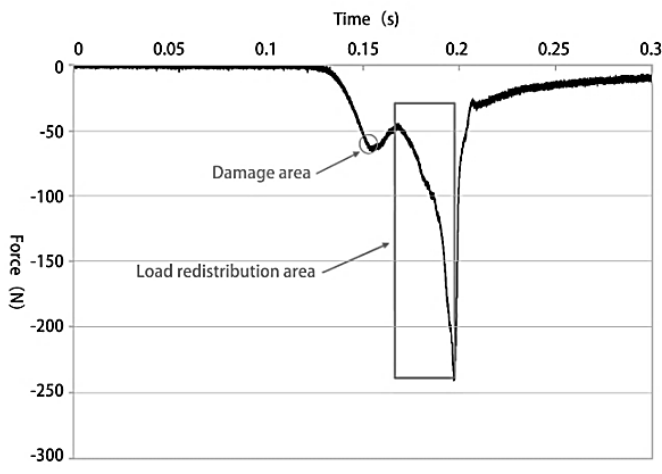

Figure 6: Typical force curve of compression sample 


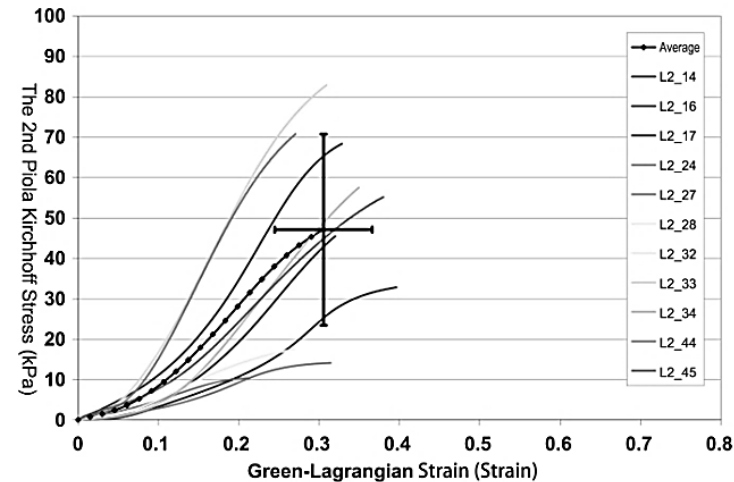

a. The liver

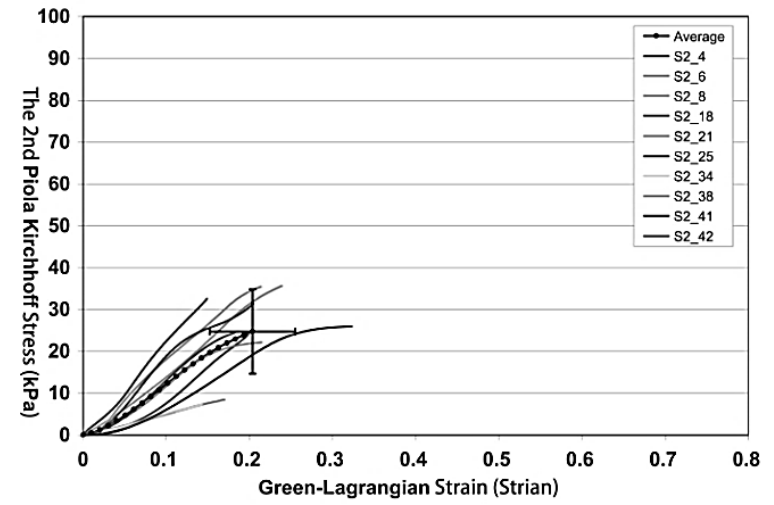

b. The spleen

Figure 7: Parenchymal response of human organs under uniaxial tensile $\sim 0.1$ and $\mathrm{S}^{-1}$

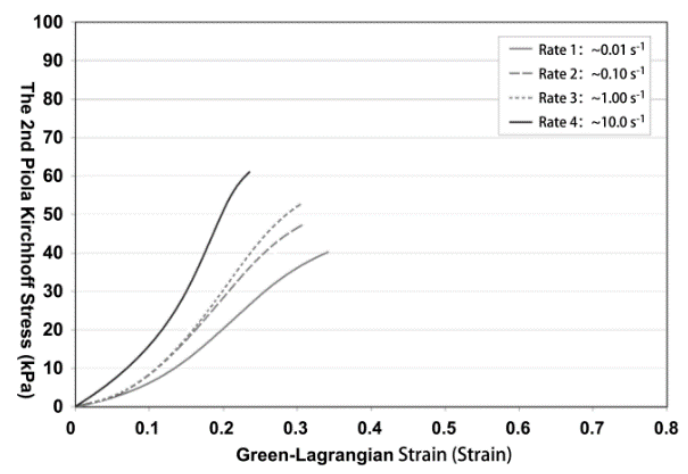

a. The liver

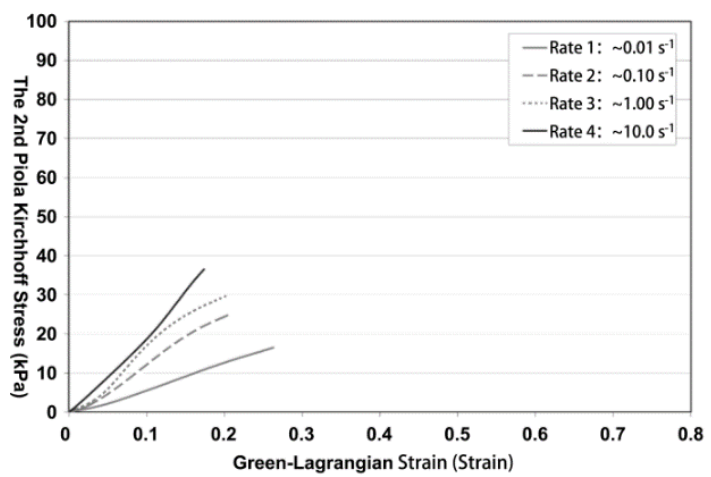

b. The spleen

Figure 8: Characteristic mean values of the parenchyma of human organs in uniaxial tension

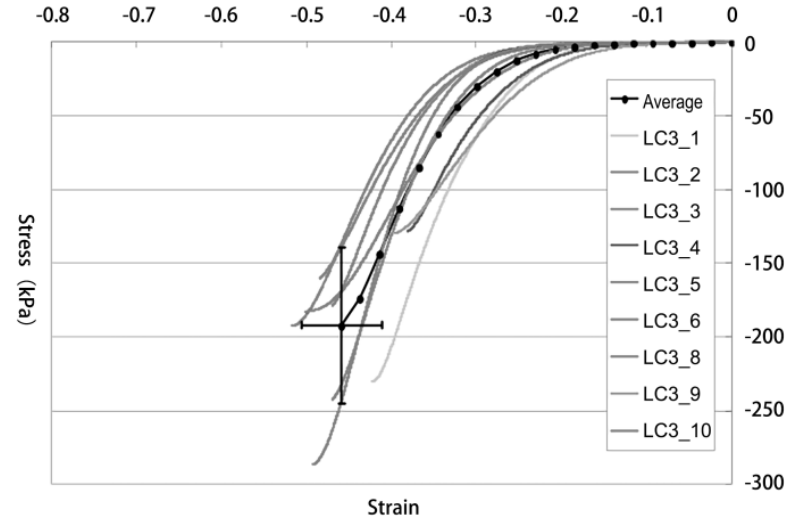

a. The liver

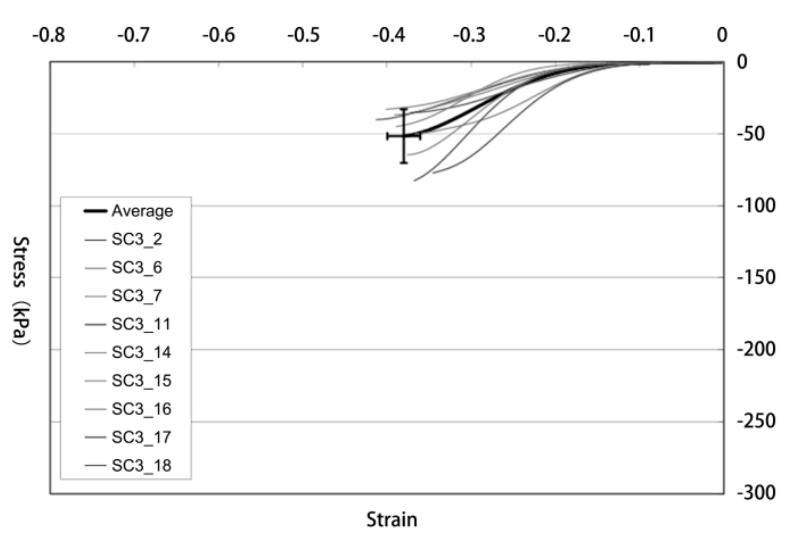

b. The spleen

Figure 9: Parenchymal response of the human organs under uniaxial compression of $\sim 0.1 \mathrm{~S}^{-1}$

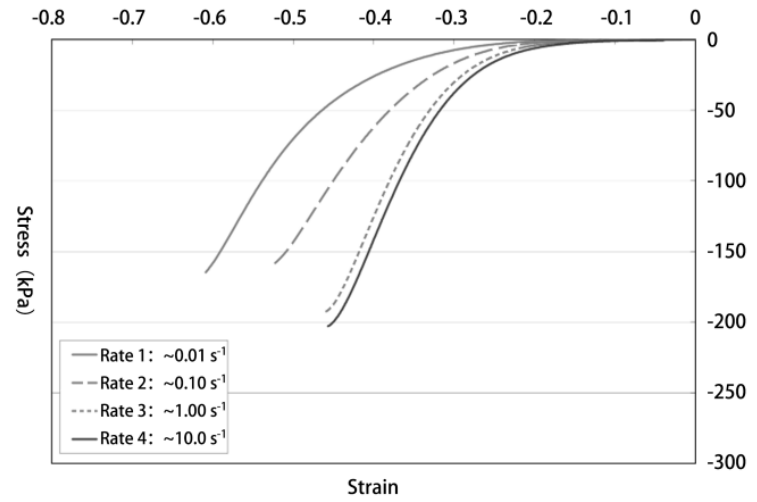

a. The liver parenchyma

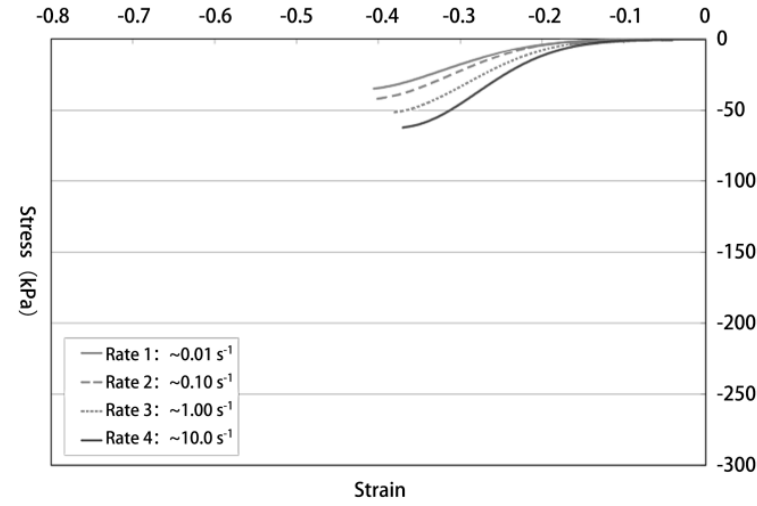

b. The spleen

Figure 10: Characteristic average value of the parenchymal compression of human organs

\section{CONCLUSION}

Cite the article: Qiu Jinlong, Wang Chunchen, Li Kui , Duan Aowen, Yin Zhiyong (2017). Biomechanical Experimental Study On Human Liver And Spleen And Optimization Discussion Of Tissue Finite Element Simulation. Topics in Intelligent Computing and Industry Design. 1(1): 48-52. 
In this study, CIREN and NASS/CDS databases have been used to analyze the overall damage. Through the whole-body organ impact test of human liver and spleen, each organ has been subjected to sub destructive test and destruction test at multiple rates. Finally, the tissue horizontal tension and compression failure tests have been then performed at each of the four different loading rates on each organ. This experiment will enhance the understanding of visceral organ damage, and provide a biomechanical basis for further establishing the finite element model of human internal organs.

\section{REFERENCES}

[1] Biswas, S., Adileh, M., Almogy, G., Miklosh Bala, M. 2014. Abdominal injury patterns in patients with seatbelt signs requiring laparotomy. Journal of emergencies, trauma, and shock, 7 (4), 295.

[2] Hardy, W.N., Howes, M.K., Kemper, A.R. 2015. Impact and Injury Response of the Abdomen. Accidental Injury. Springer New York, $373-434$ p.

[3] Beillas, P., Berthet, F. 2017. An investigation of Human Body Model morphing for the assessment of abdomen responses to impact against a population of test subjects. Traffic Injury Prevention (just-accepted), p. 00-00.

[4] Didier, C.H., Rongiéras, F., Gennisson, J.L., Philippe, B., Mickaël, T. 2016. A new method to assess the deformations of internal organs of the abdomen during impact. Traffic injury prevention,17 (8), 821-826.

[5] Ji-qing, C., Tian-ya, D., Feng-chong, L. 2017. Numerical analysis of human liver biomechanical response in blunt impacts. Journal of Jilin University (Engineering and Technology Edition) 3, 1-9. (In Chinese)

[6] Takhounts, E., Ridella, S., Tannous, R., Campbell, J., Malone, D., Danelson, K., Stitzel, J., Rowson, S., Duma, S. 2008. Investigation of Traumatic Brain Injuries Using the Next Generation of Simulated Injury Monitor (SIMon) Finite Element Head Model. Stapp Car Crash Journal, 52, 1-31.

[7] Bentil, S.A., Ramesh, K.T., Nguyen, T.D. 2016. A Dynamic Inflation Test for Soft Materials. Experimental Mechanics, 56 (5), $759-769$.

[8] Kemper, A.R., Santago, A.C., Stitzel, J.D., Sparks, J.L., Duma, S.M. 2012. Biomechanical response of human spleen in tensile loading. Journal of biomechanics 45 (2), 348-355.

[9] Gao, Z., Lister, K., Desai, J.P. 2010. Constitutive modeling of liver tissue: experiment and theory. Annals of biomedical engineering, 38 (2), 505-516.

[10] Kemper, A.R., Santago, A.C., Stitzel, J.D., Sparks, J.L., Duma, S.M. 2013. Effect of strain rate on the material properties of human liver parenchyma in unconfined compression. Journal of biomechanical engineering, 135 (10), 104-503.

[11] Thor, C. 2008. Characteristics of Thoracic Organ Injuries in Frontal Crashes. MS Thesis Virginia Polytechnic Institute and State University. 